\title{
The Learning of Chinese Idiomatic Expressions as a Foreign Language
}

\author{
$\operatorname{Li~Liu}^{1} \&$ Jiayi Yao ${ }^{2}$ \\ ${ }^{1}$ Faculty of English Education, Guangdong University of Foreign Studies, Guangzhou, China \\ ${ }^{2}$ Faculty of English Language and Culture, Guangdong University of Foreign Studies, Guangzhou, China \\ Correspondence: Li Liu, Faculty of English Education, Guangdong University of Foreign Studies, No. 2 North \\ Baiyun Road, Baiyun District, Guangzhou, China. Tel: 86-136-1144-2506. E-mail: kristenliul@hotmail.com
}

Received: March 13, 2017

Accepted: March 17, 2017

Online Published: April 5, 2017

doi:10.5539/hes.v7n2p27

URL: http://doi.org/10.5539/hes.v7n2p27

\begin{abstract}
Chinese idioms are mostly four-character phrases and are called Quadra-syllabic Idiomatic Expressions (QIEs). It has long been reported that learning of Chinese QIEs poses a great challenge for both young L1 speakers and adult L2 learners as the condensed form is often associated with complicated figurative meanings. The present study explored the factors that influence the learning of Chinese QIEs as a Foreign Language (FL). The results of a comprehension test and a questionnaire showed that semantically transparent QIEs were understood much better than opaque ones; being structurally symmetric also facilitated QIE understanding, but with limited effect. Language transfer was another factor to consider especially when the learners were from mixed nationalities. The results were then compared with those reported in the Native Language (NL) QIE learning and were further discussed in terms of the pedagogical implications for the learning of QIEs in FL teaching.
\end{abstract}

Keywords: Chinese idioms, opacity, symmetry, foreign language learning

\section{Introduction}

As Chinese becomes more and more popular as a Foreign Language (FL) around the globe, studies on the learning of Chinese have raised strong research interest. Chinese idioms, mostly composed of four characters and also called Quadra-syllabic Idiomatic Expressions (QIEs), have long been reported as a great challenge for FL learners (Cui, 2008; Zhang, 2003). The short four-word form is often associated with opaque idiomatic meanings. Yet few empirical studies have explored the learning of QIEs under the FL context so far. Therefore, it is of high necessity to understand why QIEs are so difficult for adult FL learners? Are there some kinds of QIEs more difficult than others? What might be the linguistic factors that influence QIE learning as a FL?

\subsection{Chinese QIEs}

An often-cited definition of Chinese idioms is the one from Xiandai hanyu cidan (Modern Chinese Dictionary) (1959), as cited in the following:

Idioms are set phrases and short sentences, usually in pithy forms with concise meaning, prevalent in society, used by the common folk, which have seen ages of constant use. Idioms are mostly composed of four characters. The meanings for some of them are not difficulty to deuce from the constituents, such as "xiao-ti-da-zuo: make an issue out of a minor theme", which means to fuss about trifles, "hou-lai-ju-shang: those that started late get ahead", which means "later started finish first", etc. On the other hand, with some idioms, their meanings cannot be directly gained from their constituents unless we know the semantic fields or the historical sources such as the incident, or the background from which the particular set phrase comes about. Examples of this are "zhao-san-mu-si: three in the morning and four in the evening", which means "chop and change", or "of two minds about"; "bei-gong-she-ying: the reflection of a bow in a cup a snake", which means "take very bush for a bugbear", etc. (translated in Wu, 1995, p. 63).

This definition highlights several features of Chinese idioms: structural inflexibility, semantic opacity, and their ubiquity in daily communication. Chinese idiomatic expressions display a unique cross-linguistic feature as over 95\% consist of four characters (Zhou, 1997). Hence they are also referred to as QIEs (Liu \& Cheung, 2014). The meaning of Chinese QIEs thus tends to be particularly obscure due to the short four-word format. What's more, 
the use of classical Chinese characters or cultural anecdotes may make the inference of QIE meaning even more challenging. Yet QIE use is pervasive in written and oral discourse. Some studies report that Chinese people prefer to use a greater number of idioms then westerners in their daily communication (Zhang, 2003). Owning to their elegant and prosodic style, QIEs are also frequently employed in public speeches of all kinds (Nall, 2009). Below let's look at the linguistic features of Chinese QIEs in more detail.

\subsubsection{Internal Structure of Chinese QIEs}

The structure of a QIE still has to conform to the constraints of general grammar in spite of the pithy form (Ni, 1997). Most QIEs consist of two parts: the first two morphemes are the fore part and the last two morphemes the hind part. The relation between the two parts can be various, such as Verb-noun (as in ji-zhong-yao-hai: hit-on-the-vital-point, hit the needle), adjective-noun (as in hai-qun-zhi-ma: disgrace-group-horse, the black sheep), modifier-modified (as in chen-re-da-tie: while-hot-strike-iron, take the good opportunity), etc. And each part also has its own internal structure. It is documented that for more than one third of QIEs, the two parts are symmetric in structure and semantics (Chen, 1982), that is, they have identical internal structure and have similar or opposite meanings. For example, in tian-ya-hai-jiao (sky-end-sea-corner), the fore part tian-ya (sky-end) has identical structure with the hind part hai-jiao (sea-corner), and they both refer to similar meanings: the rather remote area in the globe. Such feature of being symmetric in structure actually provides additional information in QIE meaning inference, as the two parts either reinforce or set contrasts to each other in semantics.

\subsubsection{Semantic Opacity of Chinese QIEs}

The figurative meaning of Chinese idioms varies in terms of its compositionality (Zhang, 2002). Some are more compositional and therefore transparent in meaning as they can be largely understood based on the literal meaning of each constituent, such as shi-ban-gong-bei (work-half-achieve-double). Some tend to be more non-compositional and semantically opaque as the figurative meaning involves specific knowledge of metaphor or metonymy, as in he-li-ji-qun (crane-standing-among-chickens: to stand out in the crowd). What's more, a fair proportion of Chinese idioms may contain classical Chinese and historical anecdotes, making the overall idiomatic meaning quite conventional and culture-bound. For example, the meaning of liang-shang-jun-zi (a-gentleman-on-beam) describes a well-known historical story and refers to a burglar or thief, instead of a gentleman as indicated literally.

\subsection{Learning of Chinese QIEs}

Current empirical studies on the learning and acquisition of Chinese QIEs are mainly conducted in Native Language (NL) learning. A number of factors have been identified as affecting QIE acquisition. Some of these factors are general ones as they might affect idiom learning in any languages, such as semantic transparency (Lin, 2009; Liu \& Cheung, 2014); context and familiarity (Hsieh \& Hsu, 2010); while some are of particular significance in Chinese QIE learning as they relate to their unique and salient features, such as the structural format, semantic symmetry, use of classical Chinese, etc. (Huang et al., 1999; Liu \& Xing, 2000; Liu \& Cheung, 2014). The following will introduce some of the specific studies.

Liu and Xing's survey (2000) of 35758 entries in the dictionary of Han Yu Cheng Yu Ci Hai (Chinese Idiom Dictionary) revealed that about $40 \%$ of the idioms in their data exhibit the feature of semantic symmetry (the fore part has identical, similar or opposite meaning to the hind part). Their subsequent lexical retrieval experiment indicated that idioms with semantic symmetry were much easier to memorize than those without.

Huang et al.'s study (1999) tested the effect of symmetric structure (i.e., the internal structure of the fore part and the hind part is identical in syntactic relation) and familiarity on idiom identification and re-recognition. The results indicated there was significant effect of both factors in the identification task. Response time for idioms with symmetric structure and familiar idioms were both shorter than those with no such structure and less familiar. But in the re-recognition test, significant effect was only found with structural symmetry but not familiarity.

Lin's study (2009) explored the specific effect of semantic compositionality (or transparency) on Chinese idiom comprehension. Based on the contribution individual constitutes make to the idiomatic meaning and whether background knowledge was required for comprehension, a transparency scale was first developed, which divided Chinese idioms into transparent, semi-transparent and opaque ones. Subsequent comprehension tests on 9 unfamiliar idioms showed that there was a significant effect of idiom type: transparent and semi-transparent idioms both scored significantly higher than opaque ones, while no difference was found between the first two groups. Analysis of post-test questionnaires completed by the subjects showed that background knowledge was recorded as the most frequently selected reason for comprehension difficulty. The author put forward several 
teaching implications based on the findings, such as provision of contexts during learning; elaboration of cultural background and encouragement of guessing.

Hsieh and Hsu (2010) investigated the role of context, familiarity and linguistic convention in the comprehension of idiomatic expressions by Mandarin-speaking children. Their study used two experiments (a word card task and a picture selection task followed by metapragmatic task) with 3 age groups (6-year-olds; 9-year-olds and adults) to investigate their comprehension of 7 familiar and 7 unfamiliar idiomatic phrases (such as ping-guo-lian apple-face: sweet-faced and cai-niao vegetable-bird: a-green-hand) with context and with no context. They found that familiarity was an important factor for 6 and 9 year-old children when no context was given. Context played a significant role for all three age groups during idiom comprehension. Linguistic convention entered into play at the age of 6 and the effect reached significance at 9 . Children as young as 6 , or even younger, were able to use their meta-pragmatic knowledge in understanding Chinese idioms.

Liu and Cheung (2014) tested the effects of two linguistic factors in QIE learning: semantic opacity and structural symmetry, for both children (11-year-olds) and adults via a comprehension test and a sentence-production task. The effect of semantic opacity was found significant in both tasks: transparent QIEs are easier to understand and better used for both children and adults; while the advantage of being structurally symmetric was only observed in the comprehension test and that children were found as less sensitive to the symmetric feature than adults.

These reported studies have achieved important findings in the acquisition of QIEs under the NL context and pave the way for further studies in the learning of QIEs as a FL. The present study intends to fill the research gap by conducting an empirical research on the learning of Chinese QIEs under the FL context with a focus on two linguistic factors: semantic opacity and structural symmetry. The results of the present study can be compared with those reported in Liu and Cheung's study and show us whether the factors that affect QIE learning as a NL may also affect QIE learning similarly as a FL.

\section{The Present Study}

\subsection{Research Hypotheses}

Based on the above literature review, the present study aims to investigate the effect of semantic opacity and structural symmetry during the comprehension of QIEs as a FL (Production is not included here considering the fact that use of QIEs might be too demanding for FL learners). Specifically, three hypotheses are raised.

Hypothesis One: Semantically transparent QIEs will be understood better than opaque ones for adult foreign learners;

Hypothesis Two: Structurally symmetric QIEs will be understood better than asymmetric ones for adult foreign learners;

Hypothesis Three: Semantics transparent and structurally symmetric QIEs will be the easiest to understand for adult foreign learners.

\subsection{Participants}

70 overseas students in a university in Guangzhou, China took part in the study. They had all passed HSK 5 (6 levels in HSK, a standardized language ability test for those whose mother tongue is not Chinese) and their Chinese proficiency was described by their teachers as upper-intermediate to advanced level. The participants were from different nationalities, see Table 1 for detailed information. There were 38 females and 32 males, and their average age is 22 years old.

Table 1. Nationalities of participants

\begin{tabular}{lccc}
\hline Nationality & Number of Participants & Nationality & Number of Participants \\
\hline Thailand & 20 & Japan & 1 \\
Korea & 10 & South Sudan & 1 \\
Russia & 9 & Belarus & 1 \\
Kazakhstan & 8 & The Republic of Congo & 1 \\
Indonesia & 5 & Tajikistan & 1 \\
U.S.A & 2 & Iran & 1 \\
\hline
\end{tabular}




\begin{tabular}{llcc}
\hline Uzbekistan & 2 & Guinea & 1 \\
Venezuela & 2 & Mauritius & 1 \\
Vietnam & 1 & Ukraine & 1 \\
\hline
\end{tabular}

\subsection{Materials}

80 QIEs were first selected from around 18000 idioms in Chinese Idiom Dictionary according to the following criteria: First, participants have learned all the characters in the QIEs. They are supposed to know the meaning and the form of all the composing words. Second, to control frequency, we make sure all the selected QIEs do not appear in their textbooks. Third, to reduce the influence of specific Chinese cultural information, QIEs with names of people or places are not included. For instance, in ye-gong-hao-long (Lord-Ye-love-dragon: insincere love for something) and luo-yang-zhi-gui (Luo-Yang-paper-costly: sensational sale of a new book), the names of ye-gong (Lord Ye) and Luo-yang (Luo-yang City) both bear special background information, the lack of which makes the meaning of the whole QIEs quite confusing. Such QIEs were excluded.

\subsection{Instrument}

To meansure idiom comprehension, forced choices are frequently used in current studies (Nippold \& Rudzinski, 1993; Levorato \& Cacciari, 1995). A multiple comprehension test was also adopted in this study. The test included 40 selected QIEs, 20 transparent and 20 opaque. In each semantic group, half items are symmetric and half asymmetric in structure. The meaning of each QIE was presented in three choices: one is an explanation of the literal meaning; one is the correct figurative meaning; and the third is an interfering explanation based on the literal meaning of the component morphemes. See the example of hua-bing-chong-ji (draw-cakes-allay-hunger: feed on illusions) in the following.

hua-bing-chong-ji:

A. e le de shihou, jiu hua ge bing. (literal explanation)

to draw cakes when feeling hungry

B. yong kongxiang lai ziwo anwei, meiyou shiji xiaoguo. (figurative meaning)

\section{to feed on illusion, no practical effect}

C. biyu shifen shenqi de jishu. (interfering meaning)

\section{to symbolize a magical skill}

Participants are expected to choose the correct figurative meaning for each QIE. The order of the three choices for each test item is randomly arranged.

A questionnaire was also performed after the comprehension test to further explore the exact difficulty learners from different nationalities might face in QIE learning. The questionnaire includes four questions and a list of the 40 tested QIEs. Table 2 shows the English version of these questions.

Table 2. Contents of the questionnaire

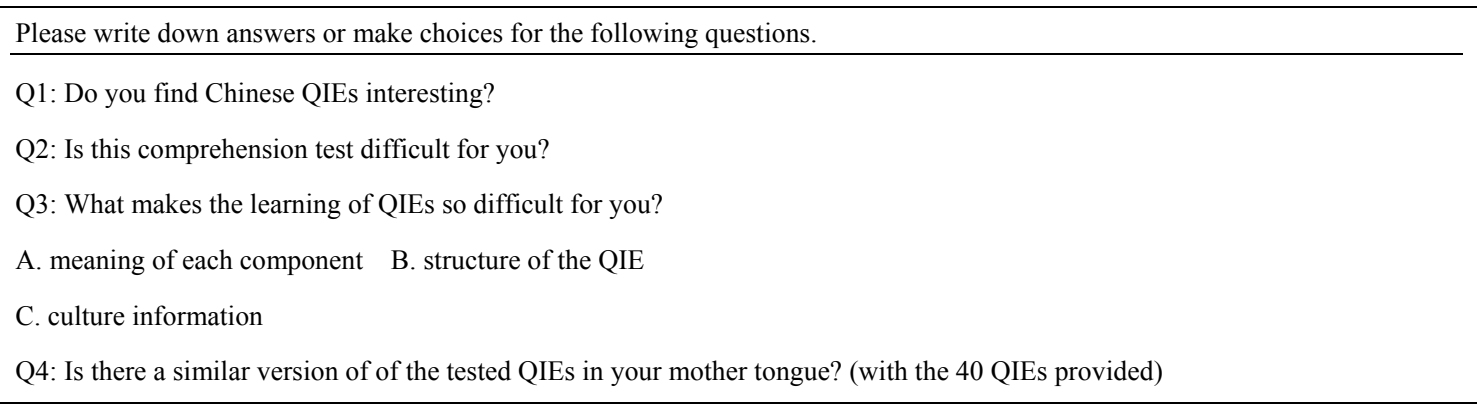




\subsection{Procedure}

Before the comprehension test, the experimenter first read the instructions slowly (Instructions: There is a large number of four-word long idioms in Chinese. They often have special figurative meanings. Please choose the correct meaning for each idiom listed below). The real test began after a practice exercise was given and every participant was clear about the requirement. It took participants 20 to 30 minutes in finishing the whole test.

Immediately after the test, participants were further invited to complete the questionnaire. They could all reply the questions in Chinese. This part took about 15 minutes.

\section{Results and Discussion}

\subsection{Results of the Comprehension Test}

10 subjects failed to finish the test and the retrieved number of effective papers was 60 . One correct choice was given 1 point. The maximum score for the test would be 40 . In addition, the number of literal and interference choices were also counted to make comparisons.

The total average score of the test was recorded as 11.12 points $(\mathrm{SD}=2.64)$. The highest score was 17 and the lowest 6 . Among the 1,200 choices, the correct choice reached up to a total of 667 , and the accuracy rate is $55.58 \%$. That means participants could understand over half of the test items. Specifically, dui-niu-tan-qin (to-ox-play-music: choose the wrong audience) got the highest correct answer rate of $87 \%$. Mu-bu-shi-ding (eyes-not-know-any-word: illiterate) also got a correction rate of $75 \%$; the lowest was found with gai-tou-huan-mian (change-appearance-alter-face: to make superficial change), with an accuracy rate of $22 \%$.

Table 3 shows participants' average correct scores according to semantic opacity and structural symmetry. The maximum score for each column is 10 points.

Table 3. Means (and SDs) of correct choices according to Opacity and Symmetry in Comprehension Test

\begin{tabular}{ccccc}
\hline & \multicolumn{2}{c}{ Opaque } & \multicolumn{2}{c}{ Transparent } \\
\hline \multirow{2}{*}{ Means } & Symmetric & Asymmetric & Symmetric & Asymmetric \\
& $2.08(0.98)$ & $3.02(0.99)$ & $3.15(1.18)$ & $2.87(1.17)$ \\
\hline
\end{tabular}

A $2 \times 2$ repeated measure of ANOVA was conducted based on the statistics in Table 3 . The results showed that the effect of semantic opacity was significant $\left(F(1,59)=12.06, p<.001\right.$, partial $\left.\eta^{2}=.17\right)$. Transparent QIEs got higher scores than opaque ones (mean scores: 6.02 vs. 5.10). The effect of structural symmetry was not significant. But the interaction of the two factors was found significant $\left(F(1,59)=6.46, p<.000\right.$, partial $\left.\eta^{2}=.099\right)$. For transparent QIEs, those with symmetric structure got higher scores, while for opaque QIEs, those with asymmetric structure scored higher. This might partly explain why the overall effect of symmetry did not reach significance as its contradictory effects observed with opaque and transparent QIEs may counterbalance each other.

These results indicate that transparent QIEs are indeed understood better than opaque ones. Hypothesis One was confirmed. The association between the literal meaning and the figurative meaning in transparent QIEs are more direct and easier to infer, which makes the comprehension much better.

On the other hand, being structurally symmetric does not facilitate the overall comprehension of QIEs, though it does show advantage on the comprehension of transparent QIEs. The similar or opposite meaning between the fore and hind part of a QIE, as in gai-tou-huan-mian (change-appearance-alter-face: to make superficial change), qu-chang-bu-duan (use-advantage-make-up-weakness: learn from others' strong points), does not reduce the difficulty learners encounter in figuring out the meaning of an opaque QIE. Hypothesis Two was partly confirmed.

The results are not, as a matter of fact, out of expectation. The effect of semantic opacity has been witnessed in the learning of idioms in many languages, such as English, French, Italian, etc. (Gibbs, 1987; Levorato \& Cacciari, 1995; Laval, 2003). Chinese QIEs as a foreign language were proved to be no exception on this point. Nevertheless, the feature of structural symmetry is unique to Chinese QIEs, and it requires the learners are quite sensitive to the internal structure, which is rather succinct and brief. Foreign learners, however, may not have developed the linguistic competence in analyzing the internal structure and realize the syntactic and semantic relation between the components of a QIE, especially for the opaque ones. Therefore, it is no surprise that the 
effect of symmetry was only observed with transparent QIEs, of which the internal structure is more self-evident and easier to analyze. The combination of transparent meaning and symmetric form leads to the best comprehension. Hypothesis 3 was confirmed.

The results reported here set contrasts with those reported in Liu and Cheung's (2014) under NL environment. In their study, the effect of opacity was significant with both adults and 11-year-olds; while the effect of symmetry was more strongly observed with adults than 11-year-olds. This implies that the effect of opacity is quite consistent in both NL and FL learning and that children and foreign learners alike are still not sensitive enough to the internal structural of a QIE.

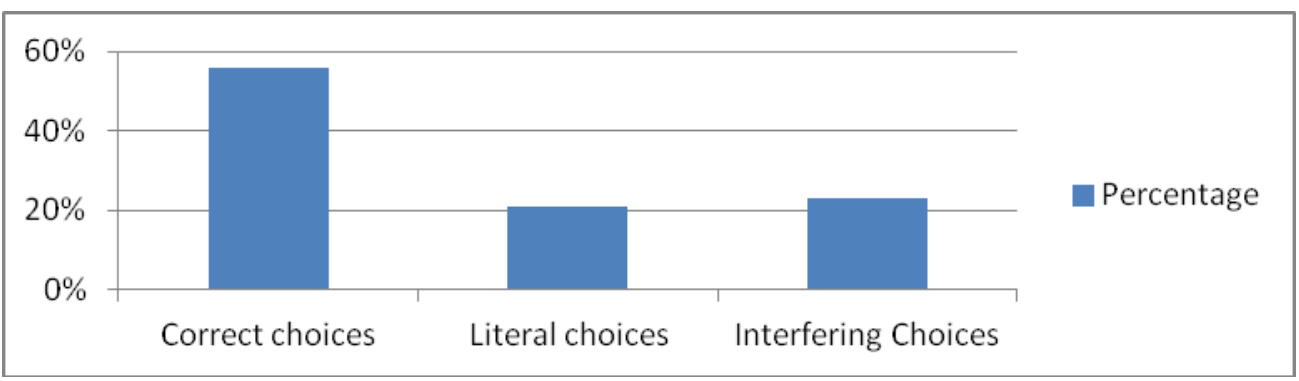

Figure 1. Percentage of participants' different choices

Finally, let's look at participants' wrong choices. There were a total of 533 wrong choices, among which 256 were literal ones and 277 interfering ones. Figure 1 shows the respective percentage of correct, literal and interfering choices. Literal choices accounted for $21 \%$ of all answers while interfering ones took $23 \%$. This shows that even though participants may not be sure about the correct figurative meaning, they would be more likely to choose the interfering meaning rather than the literal one, as they have realized that QIEs cannot be understood according to the literal meaning of each components. This preference to interfering choices rather than literal ones were more obviously observed in Liu and Cheung's study with native young speakers.

\subsection{Results of the Questionnaire}

60 copies of effective questionnaires were retrieved. It was found that over $60 \%$ of the participants acknowledged that QIEs are very interesting and learning QIEs would help them in successful communication; but at the same time, $58 \%$ of the participants indicated that QIEs are not easy to learn and the comprehension test was hard.

In regard to the factors that caused the difficulty, over half participants chose A: the meaning of each component. Over 30\% chose C: the cultural information related to the QIE. And less than $20 \%$ chose B: the structure of a QIE. This implies the in the process of QIE comprehension, meaning-related factors are more important for foreign learners than structural factors, echoing the empirical findings reported in the comprehension test. One participant even gave extra notes: only knowing the meaning of each component but not their cultural implications does not lead to successful understanding. That means foreign learners know QIEs cannot be understood on a literal basis, and the background information related to each word may be crucial in QIE comprehension.

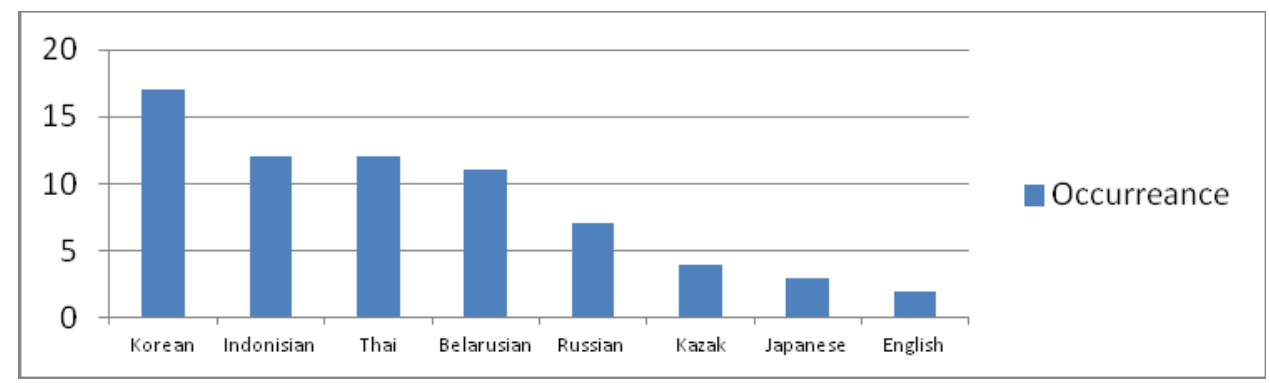

Figure 2. Occurrences of similar counterparts of tested QIEs in different languages 
A reported total of 17 tested QIEs found similar counterparts in other languages. 19 participants from 9 different countries said their languages have similar expressions of dui-niu-tan-qin (to-ox-play-music: choose the wrong audience), an item with the highest accuracy rate of $87 \%$. For example, Japanese learners reported that their language uses $d o g$ instead of $o x$ in similar expressions. Among other QIEs with high scores, some also have similar wordings in other languages, such as $m u$-bu-shi-ding (eyes-not-know-any-word: illiterate), ji-bu-ze-shi (hungry-not-pick-on-food: hunger is the best sauce), bai-nian-bu-yu (hundred-years-not-occur: once in a century), etc. See Figure 2 for details. The probability of having similar counterparts in a language seems to correlate with the geographical location of that country: the occurrence of similar counterparts decreases with the increase of geographical distance between that country and China. From Figure 2, we can see that Korean, Thai and Indonesian showed the highest frequency of having similar expressions to the tested QIEs. Other languages with high frequency include Russian and Belarus in the European area; whereas languages spoken in the African countries rarely have similar expressions.

In brief, data in the questionnaire indicate that the process of QIE comprehension and learning is also influenced by language transfer (Irujo, 1986; Charteris-Black, 2002). Understanding of QIEs can be greatly enhanced when there is identical or similar counterparts of a QIE in their own language. The closer a country locates to China, the higher the probability that such counterparts are available in its language.

\section{Summary and Conclusion}

This study presents an empirical exploration on the comprehension of Chinese QIEs for FL learners. Specifically, it examines the effects of two linguistic factors: semantic opacity and structural symmetry. Findings of the study indicate that semantic opacity plays an important role in QIE comprehension: foreign learners understand transparent QIEs much better; the positive role of being structurally symmetric is, nonetheless, restricted to transparent QIEs. Findings also show that foreign learners understand the QIEs that are simultaneously transparent and symmetric the best.

These findings of QIE learning are actually quite consistent with those reported in the NL environment. In both the FL and NL contexts, the effect of opacity is similarly observed. Regarding the effect of symmetry, FL learners behaved similarly with native 11-year-olds, however. Their Chinese competence has not become mature enough to enable them to notice and analyze the internal structure of a QIE.

Under the NL context, QIEs that are opaque and asymmetric are found to pose the most difficulty, which was not observed here under the FL context. The reason might be related with language transfer. As indicated in the questionnaire results, several test items in the comprehension test were self-reported by the participants as having similar counterparts in their own language, such as dui-niu-tan-qin and mu-bu-shi-ding, which are opaque and asymmetric test items in this study. The effect of positive transfer greatly increased the correct understanding of such QIEs, partly explaining why no identical results were observed here in the FL context.

Findings in this study might shed some lights on the pedagogy of Chinese QIE teaching. It is not necessary that teachers sacrifice QIE use in teaching materials or classroom talk for easy communication, as foreign learners generally show great interest in QIE learning. To explain the meaning of each component (sometimes background information) is preferred than asking students to memorize the QIE as a chunk since knowing the meaning of individual components is the major strategy they use in the meaning inference. Ideally, knowing the mother tongues of foreign learners would be a great advantage for teachers in that they may start from those similar expressions and gradually draw learners' attention to the different ones.

With the population of FL learners of Chinese are increasing worldwide, it is of high necessity to study the learning process of Chinese as a FL and investigate on the factors that affect this process. Learning of Chinese QIEs is an indispensable part, especially for the advanced learners, as QIEs are widely used in daily communication. The present study focused on the linguistic factors and obtained some useful findings in QIE learning. However, it still has several limitations. First, due to practical reasons, the language backgrounds of participants were not controlled. Some of the languages exhibited more probability of positive transfer than others, making the test results hard to explain. In addition, apart from the linguistic factors, future studies should try to look at the effect of context in QIE comprehension (Cooper, 1999). Finally, the results reported here can be re-examined with learners of more advanced Chinese proficiency.

\section{Acknowledgements}

This research is supported by a grant from the Ministry of Education in P.R.C., given to the first author (15YJC740048). Special thanks are given to the overseas students who voluntarily took part in this research. 


\section{References}

Charteris-Black, J. (2002). Second language figurative proficiency: A comparative study of Malay and English. Applied Linguistics, 23(1), 104-133. https://doi.org/10.1093/applin/23.1.104

Chen, W. B. (1982). English idioms and Chinese Chengyu. Beijing: Foreign Language teaching and research Press.

Cooper, T. C. (1999). Processing of idioms by L2 learners of English. TESOL Quarterly, 33(2), 233-262. https://doi.org/10.2307/3587719

Cui, X. L. (2008). Cognitive and learning of Chinese as a second language. Beijing: Beijing University Press.

Gibbs, R. W. (1987). Linguistic factors in children's understanding of idioms. Journal of Child Language, 14, 569-586. https://doi.org/10.1017/S0305000900010291

Hsieh, S. C. Y., \& Hsu, C. C. N. (2010). Idiom comprehension in Mandarin-speaking children. Journal of Psycholinguist Research, 39, 505-522. https://doi.org/10.1007/s10936-009-9145-z

Huang, X. T., Chen, W. F., Yu, H., \& Wang, W. H. (1999). A research on the cognition of symmetrical structural phrases in Chinese. Psychological Sciences, 3, 193-196.

Irujo, S. (1986). Don't put your leg in your mouth: Transfer in the acquisition of idioms in a second language. TESOL QUARTERLY, 20(2), 287-304. https://doi.org/10.2307/3586545

Laval, V. (2003). Idiom comprehension and metapragmatic knowledge in French children. Journal of Pragmatics, 35(5), 723-739. https://doi.org/10.1016/S0378-2166(02)00137-6

Levorato, M. C., \& Cacciari, C. (1995). The effects of different tasks on the comprehension and production of idioms in children. Journal of Experimental Child Psychology, 60(2), 261-283. https://doi.org/10.1006/jecp.1995.1041

Lin, C. J. (2009). What makes Chinese idioms so difficult to understand? A study of the semantic transparency of Chinese idioms. Chinese Teaching and Research, 6(2), 163-188.

Liu, L., \& Cheung, H. T. (2014). Acquisition of Chinese quadra-syllabic idiomatic expressions: Effects of semantic opacity and structural symmetry. First Language, 34(4), 336-353. https://doi.org/10.1177/0142723714544409

Liu, Z. Q., \& Xing, M. P. (2000). A cognitive study on Chinese four-character idioms with semantic symmetric features. Chinese Teaching in the World, 1, 77-81.

Nall, T. (2009). An Analysis of Chinese Four-Character Idioms Containing Numbers: Structural Patterns and Cultural significance (Unpublished PhD Thesis). Ball State University.

Ni, B. Y., \& Yao, P. C. (1997). Cheng yu bian xi ci dian [Idioms with Similar Characters]. Beijing: The Commercial Press.

Nippold, M. A., \& Rudzinski, M. (1993). Familiarity and transparency in idiom explanation: A developmental study of children and adolescence. Journal of Speech, Language and Hearing Research, 36, 728-737. https://doi.org/10.1044/jshr.3604.728

Tsou, B. K. (2012). Idiomaticity and classical traditions in some East Asian languages. Paper presented at the the 26th Pacific Asia Conference on Language, Information, and Computation. Retrieved from http://aclweb.org/anthology/Y/Y12/Y12-1003.pdf

Wu, Chu-hsia. (1995). On the cultural traits of Chinese idioms. Intercultural Communication Studies, 1, 61-81.

Zhang, H. (2003). Idioms and their comprehension: A cognitive semantic perspective. Beijing: Military Yiwen Press.

Zhang, B. (2002). Xin bian xian dai han yu [New Modern Chinese]. Shanghai: Fudan University Press.

Zhou, J. (1997). On the Classic feature on Chinese idioms. Journal of NanKai University, 2, 29-51.

\section{Copyrights}

Copyright for this article is retained by the author(s), with first publication rights granted to the journal.

This is an open-access article distributed under the terms and conditions of the Creative Commons Attribution license (http://creativecommons.org/licenses/by/4.0/). 